\title{
Salicylic Acid Inhibits the Replication of Tomato bushy stunt virus by Directly Targeting a Host Component in the Replication Complex
}

\author{
Miaoying Tian, ${ }^{1}$ Zsuzsanna Sasvari, ${ }^{2}$ Paulina Alatriste Gonzalez, ${ }^{2}$ Giulia Friso, ${ }^{3}$ Elden Rowland, ${ }^{3}$ \\ Xiao-Min Liu, ${ }^{1}$ Klaas J. van Wijk, ${ }^{3}$ Peter D. Nagy, ${ }^{2}$ and Daniel F. Klessig' \\ ${ }^{1}$ Boyce Thompson Institute for Plant Research, Ithaca, NY 14853, U.S.A.; ${ }^{2}$ Department of Plant Pathology, University of \\ Kentucky, Lexington 40546, U.S.A.; ${ }^{3}$ Department of Plant Biology, Cornell University, Ithaca, NY 4853, U.S.A.
}

Submitted 5 September 2014. Accepted 24 November 2014.

\begin{abstract}
Although the plant hormone salicylic acid (SA) plays a central role in signaling resistance to viral infection, the underlying mechanisms are only partially understood. Identification and characterization of SA's direct targets have been shown to be an effective strategy for dissecting the complex SA-mediated defense signaling network. In search of additional SA targets, we previously developed two sensitive approaches that utilize SA analogs in conjunction with either a photoaffinity labeling technique or surface plasmon resonance-based technology to identify and evaluate candidate SA-binding proteins (SABPs) from Arabidopsis. Using these approaches, we have now identified several members of the Arabidopsis glyceraldehyde 3-phosphate dehydrogenase (GAPDH) protein family, including two chloroplast-localized and two cytosolic isoforms, as SABPs. Cytosolic GAPDH is a well-known glycolytic enzyme; it also is an important host factor involved in the replication of Tomato bushy stunt virus (TBSV), a single-stranded RNA virus. Using a yeast cell-free extract, an in vivo yeast replication system, and plant protoplasts, we demonstrate that SA inhibits TBSV replication. SA does so by inhibiting the binding of cytosolic GAPDH to the negative (-)RNA strand of TBSV. Thus, this study reveals a novel molecular mechanism through which SA regulates virus replication.
\end{abstract}

To defend against fungal, bacterial, and oomycete pathogens, plants have evolved a multilayer immune system comprising pathogen-associated molecular pattern (PAMP)-triggered immunity (PTI), effector-triggered immunity (ETI), and a wholeplant immunity termed systemic acquired resistance (SAR) (Chisholm et al. 2006; Dempsey and Klessig 2012; Durrant and Dong 2004; Jones and Dangl 2006). Many studies have shown that salicylic acid (SA) serves as a key signal molecule for activating PTI, ETI, and SAR following infection by these diverse types of pathogens (An and Mou 2011; Vlot et al. 2009). In comparison, the mechanism through which plants resist viral infection is less well understood. Because viruses are intracellular

Current address for M. Tian: Department of Plant and Environmental Protection Sciences, University of Hawaii at Manoa, Honolulu 96822, U.S.A.

Current address for: X.-M. Liu: Michael Smith Laboratories, University of British Columbia, Vancouver, BC V6T 1Z4, Canada.

Corresponding author: D. F. Klessig; Telephone: +1.607.254.4560; Fax: +1.607.254.6779; E-mail:dfk8@cornell.edu

๑) 2015 The American Phytopathological Society pathogens and do not generate extracellular PAMPs, they may not trigger PTI, which is activated via PAMP recognition receptors with extracellular recognition or binding domains (Carr et al. 2010). However, a number of plant resistance (R) proteins that recognize specific viruses have been identified. Like many of the $\mathrm{R}$ proteins that recognize bacterial, fungal, and oomycete pathogens, the antiviral $\mathrm{R}$ proteins belong to the nucleotidebinding site leucine-rich repeat (NBS-LRR) class, and they trigger ETI and SAR via SA-dependent pathways that appear identical to those activated by nonviral pathogens (Baebler et al. 2014; Sánchez et al. 2010; Soosaar et al. 2005; Takahashi et al. 2002; Vlot et al. 2009).

In addition to these $\mathrm{R}$ protein-mediated resistance responses, plants employ RNA silencing to combat viral infection. Activation of this cellular process by viral-derived double-stranded RNAs leads to the targeted destruction of transcripts generated by both RNA and DNA viruses (Carr et al. 2010; Soosaar et al. 2005). The combined observations that (i) expression of RNAdependent RNA polymerase 1, a critical component of the RNA silencing machinery, is induced by SA treatment in a variety of plants (Liu et al. 2009; Quilis et al. 2008; Xie et al. 2001; Yang et al. 2004; Yu et al. 2003) and (ii) SA-induced gene expression is suppressed by certain viral silencing suppressor proteins (Alamillo et al. 2006; Ji and Ding 2001) suggest that there is at least some level of cross-talk between RNA silencing and the SA-inducible resistance pathways. Other studies have suggested that resistance to various viruses but not bacteria or fungi is mediated via SA-induced activation of the mitochondrial alternative respiratory pathway (Carr et al. 2010; Chivasa et al. 1997; Murphy et al. 1999; Singh et al. 2004), although the mechanism remains to be determined (Carr et al. 2010; Singh et al. 2004). Together, these findings suggest that SA's ability to suppress all three major stages of virus infection, including replication, cellto-cell movement, and long-distance movement (Murphy et al. 1999; Singh et al. 2004), is dependent on its parallel activation of several antiviral resistance mechanisms.

To gain insights into how SA exerts its many functions, a variety of SA effectors and target proteins have been identified. In addition to catalase (Chen et al. 1993), ascorbate peroxidase (Durner and Klessig 1995), chloroplast carbonic anhydrase (Slaymaker et al. 2002), methyl salicylate esterase (Kumar and Klessig 2003; Vlot et al. 2008), NPR1 (Wu et al. 2012), and its homologs NPR3 and NPR4 (Fu et al. 2012), two new highthroughput screens have identified several more SA-binding proteins (SABPs), including the E2 subunit of $\alpha$-ketoglutarate dehydrogenase (Tian et al. 2012); the glutathione $S$-transferases GSTF2, GSTF8, GSTF10, and GSTF11 (Tian et al. 2012); and 
the oligopeptidases TOP1 and TOP2 (Moreau et al. 2013). In addition, here, we report that several isoforms of glyceraldehyde 3-phosphate dehydrogenase (GAPDH), an abundant enzyme conserved in all organisms (Sirover 1999), are SABPs. Although GAPDH was first recognized for its enzymatic role in glycolysis, it was subsequently found to be involved in diverse nonmetabolic processes such as transcription activation, apoptosis, membrane fusion, DNA replication and repair, and nuclear RNA export (Sirover 1999, 2005; Tristan et al. 2011). In addition, GAPDH was shown to bind RNAs from a range of viruses (De et al. 1996; Petrik et al. 1999; Schultz et al. 1996; Wang and Nagy 2008; Zang et al. 1998).

Tomato bushy stunt virus (TBSV) is a single, positive (+)-strand RNA virus that infects a wide range of plants (Huang and Nagy 2011; Wang and Nagy 2008; White and Nagy 2004). Like other (+)RNA viruses, TBSV replication occurs in the cytosol and is directed by a membrane-bound replicase complex consisting of both viral-encoded proteins and co-opted host proteins (Huang and Nagy 2011; Nagy and Pogany 2012). A proteomics analysis of the TBSV replicase revealed that one of these co-opted host proteins is GAPDH (Serva and Nagy 2006). Subsequent
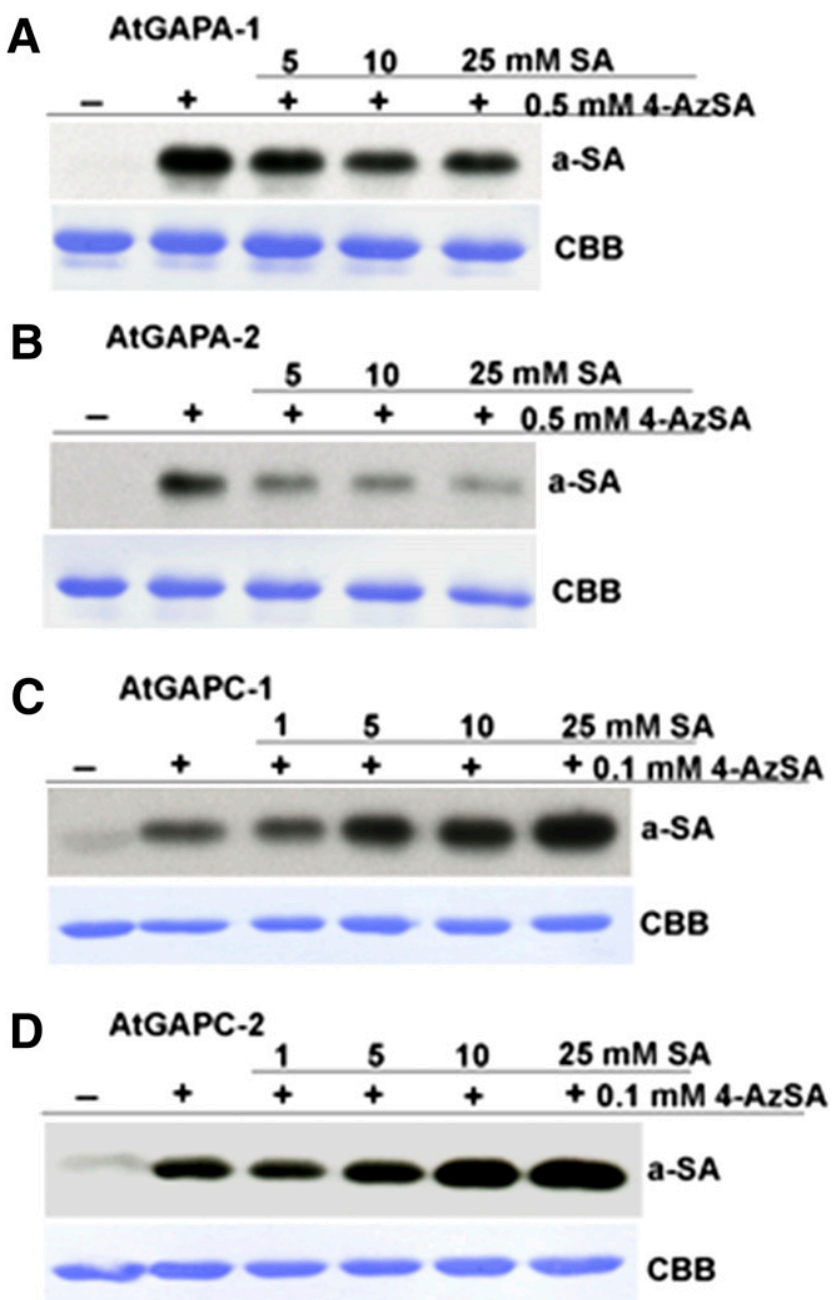

Fig. 1. Salicylic acid (SA)-binding activity of several AtGAPDH isoforms was detected by photoaffinity labeling with 4-AzSA. Purified recombinant proteins A, AtGAPA-1; B, AtGAPA-2; C, AtGAPC-1; and D, AtGAPC-2 were incubated with $(+)$ or without $(-) 4-\mathrm{AzSA}$ in the absence or presence of the indicated concentrations of SA on ice for $1 \mathrm{~h}$ and then exposed to UV light $(50 \mathrm{~mJ})$. Detection of 4-AzSA-crosslinked proteins was performed using immunoblot analysis with an $\alpha-S A$ antibody. Proteins stained with Coomassie Brilliant Blue R-250 (CBB) served as a loading control. studies indicated that GAPDH binds the (-)RNA strand of TBSV with greater efficiency than the $(+)$ strand, and this binding is required for asymmetric TBSV replication, in which higher levels of (+)RNA progeny are synthesized by the viral replicase compared with the (-)RNA replication intermediate (Huang and Nagy 2011; Wang and Nagy 2008). Given GAPDH's role in TBSV replication and its identification as an SABP, we tested whether SA affects TBSV replication via its interaction with GAPDH. Here, we demonstrate that GAPDH binds SA and this binding suppresses TBSV replication in vitro and in vivo in yeast cells and Nicotiana benthamiana protoplasts by inhibiting the binding of GAPDH to the viral (-)RNA strand, thereby revealing a novel molecular mechanism through which SA mediates viral replication.

\section{RESULTS}

Identification and characterization

of Arabidopsis GAPDH isoforms as SA-binding proteins.

We previously developed a sensitive photoaffinity labeling approach using the photoreactive SA analog 4-azido SA (4-AzSA) as a probe to identify candidate SABPs (cSABPs) from Arabidopsis (Tian et al. 2012). From a total of four pull-down assays, several dozen cSABPs were identified, including several members of the GAPDH family. In Arabidopsis, this family is comprised of seven members (The Arabidopsis Information Resource) (Muñoz-Bertomeu et al. 2009), among which we identified AtGAPA-1 (At3g26650), AtGAPA-2 (At1g12900), AtGAPB (At1g42970), AtGAPC-1 (At3g04120), and AtGAPC-2 (At1g13440) as cSABPs. AtGAPA-1, AtGAPA-2, and AtGAPB are chloroplast localized and involved in the photosynthetic reductive carbon cycle, while AtGAPC- 1 and AtGAPC- 2 are cytosolic glycolytic enzymes.

To validate the SA-binding activity of these five AtGAPDHs, we expressed and purified their recombinant, his-tagged proteins from Escherichia coli. Two sensitive approaches that utilize SA analogs in conjunction with either a photoaffinity labeling technique or a surface plasmon resonance (SPR)-based technology (Tian et al. 2012) were used for the validation. For the photoaffinity labeling approach, recombinant AtGAPDHs were crosslinked with 4-AzSA by UV irradiation in the presence or absence of SA (adjusted to $\mathrm{pH} 7.4$ ), followed by immunoblot analysis with anti-SA antibody ( $\alpha-S A$ Ab). AtGAPA-1 and AtGAPA-2 crosslinked with 4-AzSA, and the crosslinking was inhibited in the presence of excess SA, confirming their SA-binding activity (Fig. 1A and B). AtGAPC-1 and AtGAPC-2 also crosslinked with 4-AzSA (Fig. 1C and D). Interestingly, crosslinking was enhanced in the presence of increasing concentrations of SA in a dose-dependent manner. This result may suggest that AtGAPC-1 and AtGAPC-2 contain two or more SAbinding sites which interact allosterically, such that binding of $\mathrm{SA}$ at one site enhances subsequent binding at the other site or sites. Although AtGAPB also crosslinked with 4-AzSA, this crosslinking was not altered by the presence of SA (data not shown). In summary, crosslinking of AtGAPA-1, AtGAPA-2, AtGAPC-1, and AtGAPC-2 to 4-AzSA was either suppressed or enhanced by the presence of SA, suggesting that their interaction with 4-AzSA represents authentic SA-binding activity.

SA-binding activity was further confirmed for these four GAPDH isoforms using the SPR-based approach developed previously (Tian et al. 2012). When AtGAPA-1 and AtGAPA-2 were passed over a CM5 sensor chip surface to which the SA analog 3-aminoethyl SA (3-AESA) was immobilized, binding signals were observed (Fig. 2). Preincubation with SA reduced these signals in a dose-dependent manner, indicating that the ability of these proteins to bind 3-AESA was inhibited (Fig. 2A and C). Preincubation with the biologically inactive SA analog 
4-hydroxybenzoic acid (4-HBA) either failed to reduce the binding signal or reduced it to a lesser extent than comparable levels of SA (Fig. 2B and D), further suggesting that binding of AtGAPA-1 and AtGAPA-2 to the chip is a true reflection of their SA-binding activity. SPR analysis also detected binding when AtGAPC-1 or AtGAPC-2 was passed over the CM5 sensor chip surface, but the effect of SA on this binding was more complex (Fig. 3). When these proteins were preincubated with SA, binding to 3-AESA was enhanced in the presence of increasing concentrations of SA (Fig. 3A and D); this result is consistent with those obtained using the photoaffinity labeling approach. However, when the preincubation step was skipped and AtGAPC-1 or AtGAPC-2 was passed over the chip in the presence of various concentrations of $\mathrm{SA}$, the binding signal decreased as the SA concentration increased (Fig. 3B and E). Under this assay condition, 4-HBA was a less effective competitor than SA for 3-AESA binding (Fig. 3C and F). The results from these latter assays lacking the preincubation step are consistent with the hypothesis that AtGAPC- 1 contains multiple SA bindings sites but that SA binding to the first site is slow, whereas SA binding to an additional site or sites is fast. Thus, in the absence of a preincubation step, SA is able to compete with 3-AESA for binding to the "fast" binding site in AtGAPC-1. By contrast, a preincubation with SA fills the initial binding site and, thereby, enhances binding of 3-AESA in the second, "fast" binding site.

\section{SA inhibits the replication of TBSV.}

Cytosolic GAPDHs in yeast and $N$. benthamiana were shown to be required for efficient TBSV replication (Huang and Nagy 2011; Wang and Nagy 2008). Because several isoforms of AtGAPDH are SABPs, we tested whether SA affects TBSV replication using a yeast in vivo replication system, plant protoplasts, and a yeast cell-free extract (CFE) replication assay established previously (Huang and Nagy 2011; Pogany et al. 2008). In the yeast in vivo assay, $300 \mu \mathrm{M}$ SA (Fig. 4B) and $1 \mathrm{mM}$ SA (Fig. 4A) inhibited the accumulation of TBSV DI-72 replicon RNA (repRNA) by 50 and $70 \%$, respectively, whereas little to no inhibition was observed in the presence of 4-HBA (Fig. 4). Similarly, the accumulation of full-length TBSV genomic RNA and subgenomic RNAs was inhibited by SA up to approximately $90 \%$ in $N$. benthamiana protoplasts (Fig. 5). SA also inhibited repRNA accumulation in the yeast CFE replication assay. In the presence of $2 \mathrm{mM} \mathrm{SA}$, TBSV replication was strongly suppressed; greater SA concentrations led to an almost complete loss of TBSV replication (Fig. 6). By contrast, $2 \mathrm{mM}$ 4-HBA did not substantially affect TBSV replication (Fig. 6).

\section{SA inhibits the binding of AtGAPC-1}

to the (-)RNA strand of the TBSV DI-72 repRNA.

It was previously proposed that GAPDH enhances TBSV replication by selectively binding the (-)RNA and retaining it
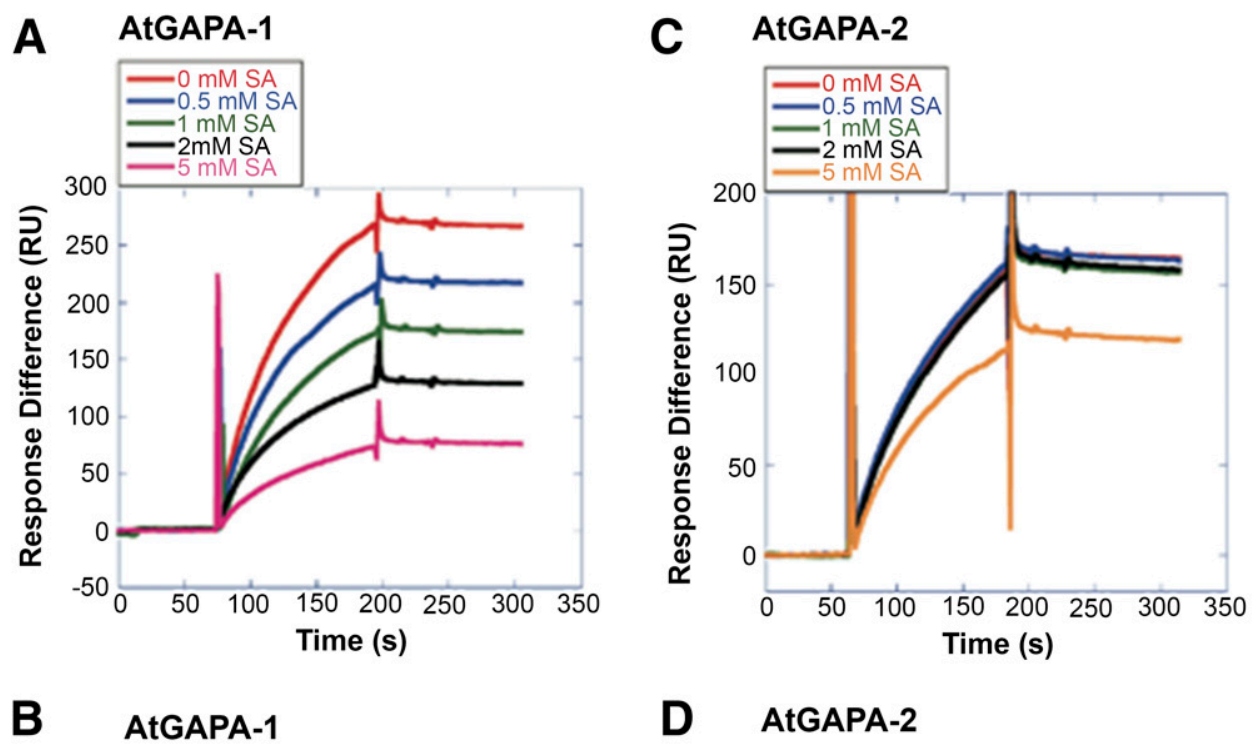

D AtGAPA-2
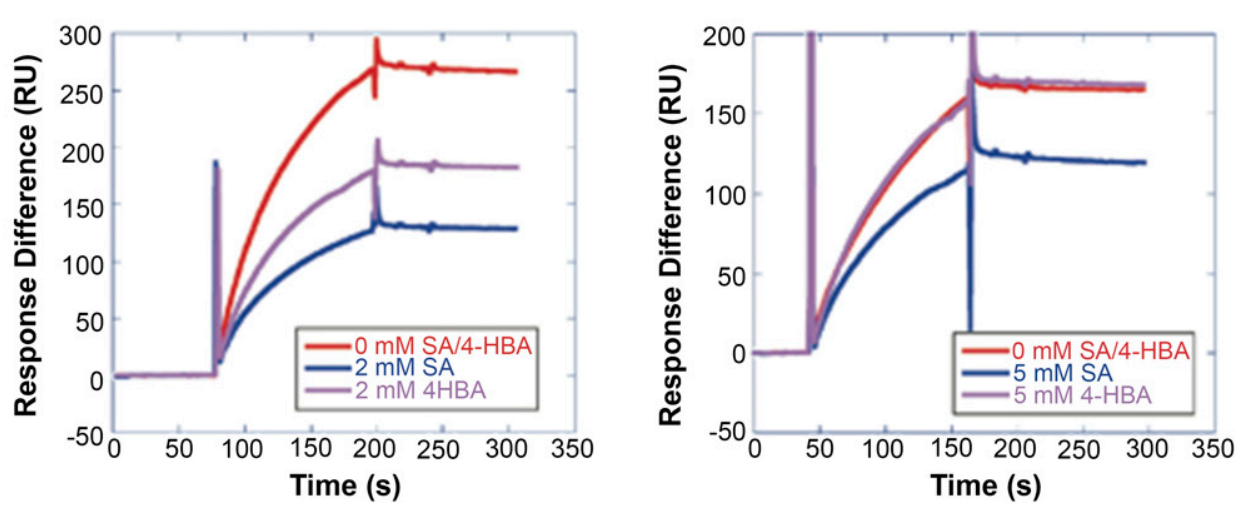

Fig. 2. Analysis of salicylic acid (SA)-binding activities of AtGAPA-1 and AtGAPA-2 by surface plasmon resonance. Sensograms of A and B, AtGAPA-1 $(1 \mu \mathrm{M})$ and $\mathbf{C}$ and $\mathbf{D}$, AtGAPA-2 $(1 \mu \mathrm{M})$ preincubated with the indicated concentrations of SA, 4-hydroxybenzoic acid (4-HBA), or equivalent volumes of $1 \times$ phosphate-buffered saline buffer on ice for $1 \mathrm{~h}$ before being passed over the 3 -AESA-immobilized sensor chip surface. The specific binding signal was determined by subtracting the signal generated with the control flow cell from the signal generated with the 3-AESA-bound flow cell. 
in the replication complex, thereby promoting multiple rounds of genomic (+)RNA synthesis (Wang and Nagy 2008).

Thus, one possible mechanism through which SA might suppress TBSV replication in the yeast in vivo and CFE assays is by disrupting the binding between GAPDH and the (-)repRNA, To assess this possibility, SPR analysis was performed using His-tagged AtGAPC-1 immobilized on an NTA chip. The validity of this approach was first tested by monitoring binding when either the (-) or (+)RNA strand of DI-72 was passed over the sensor chip surface containing immobilized His-AtGAPC-1. Consistent with prior results (Wang and Nagy 2008), the binding of AtGAPC-1 to DI-72(-) was much stronger than its binding to DI-72(+) (Fig. 7A). To test whether SA affects the binding of AtGAPC-1 to DI-72(-), $1 \mu \mathrm{M}$ of DI-72(-) was passed over the AtGAPC-1-immobilized NTA chip in the presence of increasing concentrations of SA or 4-HBA. SA suppressed GAPDH binding to DI-72(-) in a dose-dependent manner (Fig. 7B), while 4-HBA was less effective than SA at disrupting this binding (Fig. 7C).

\section{DISCUSSION}

SA has been proposed to mediate plant resistance to viral infection by activating multiple defense pathways in parallel (including signaling ETI and SAR), promoting RNA silencing, and activating the mitochondrial alternative respiration pathway (Carr et al. 2010; Singh et al. 2004; Soosaar et al. 2005; Vlot et al. 2009). In this study, we report a novel molecular mechanism through which SA confers virus resistance; it directly targets cytosolic GAPDH, a host factor that is required for TBSV replication. Plus-stranded RNA viruses rely heavily on multiple host factors to support their infection and replication (Nagy and Pogany 2012). Thus, the ability of plants to prevent the co-opting of these proteins could be a valuable antiviral strategy. Indeed, the observation that replication of Cucumber necrosis virus, a tombusvirus closely related to TBSV, was reduced in $G A P D H-C$-silenced $N$. benthamiana (Wang and Nagy 2008), is consistent with the possibility that SA targeting of GAPDH-C helps mediate resistance to this family of viruses. It should be noted, however, that $G A P D H-C$-silenced $N$. benthamiana plants support wild-type levels of replication for Tobacco mosaic virus, which belongs to a different super group of (+)RNA viruses and is unrelated to tombusviruses (Wang and Nagy 2008). Thus, modulation of GAPDH-C by SA does not appear to be a global antiviral mechanism. However, SA interacts with many diverse targets (Tian et al. 2012), and it is possible that SA mediates resistance to different subgroups of viruses by targeting other host factors required for their replication. More research will be needed to establish whether modulation of host factors represents yet another common mechanism through which SA mediates antiviral defenses.

The function of cytosolic GAPDHs in glycolysis is conserved from prokaryotes to eukaryotes. In addition, cytosolic GAPDH's RNA binding activity and its function in TBSV replication appear to be conserved. Using a yeast in vivo system, Wang and Nagy (2008) found that TBSV replication was suppressed in yeast deficient in the cytosolic GAPDHs TDH2 and TDH3. Expression of AtGAPDH-C in these TDH2- or TDH3-deficient yeast restored TBSV replication, suggesting that the cytosolic GAPDHs from yeast and Arabidopsis are interchangeable in supporting TBSV replication. The cytosolic GAPDH promotes TBSV replication through binding to an AU-rich sequence of a $3^{\prime}$ proximal region in the (-)-strand RNA and brings the TBSV $\mathrm{p} 92^{\mathrm{pol}}$ replication protein to the promoter region to facilitate initiation of (+)-strand synthesis (Huang and Nagy 2011; Kovalev et al. 2012; Wang and Nagy 2008). Thus, the ability of the yeast TDH2 protein to
A AtGAPC-1 Preincubation

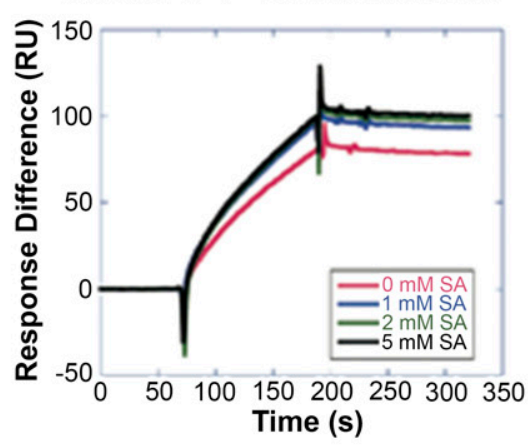

D AtGAPC-2 Preincubation

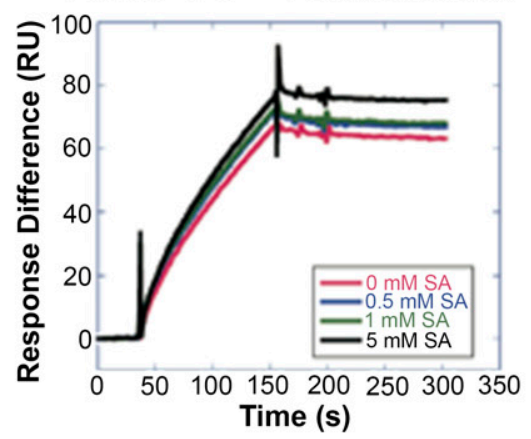

B AtGAPC-1 No preincubation

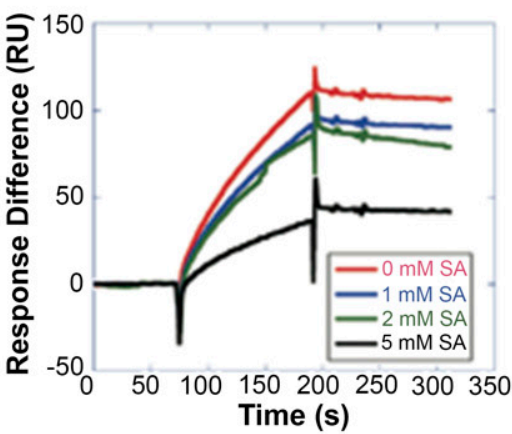

E AtGAPC-2 No preincubation

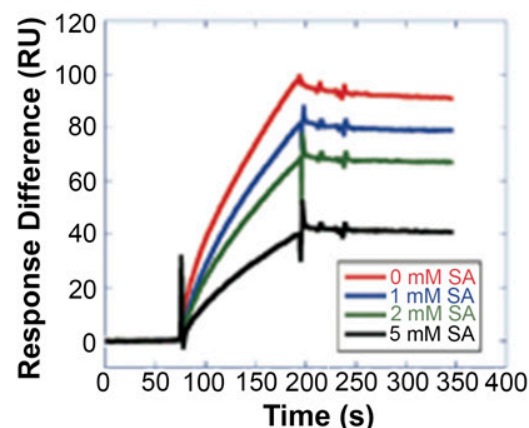

C AtGAPC-1 No preincubation

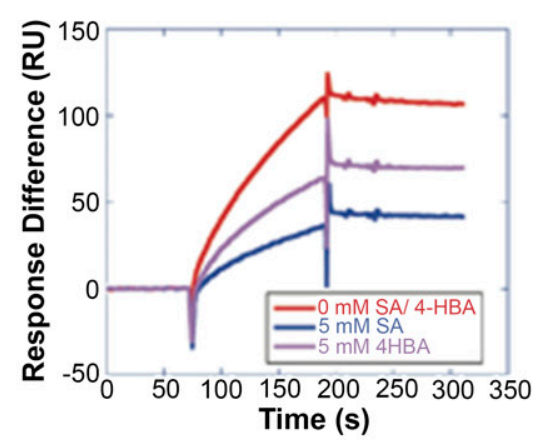

F AtGAPC-2 No preincubation

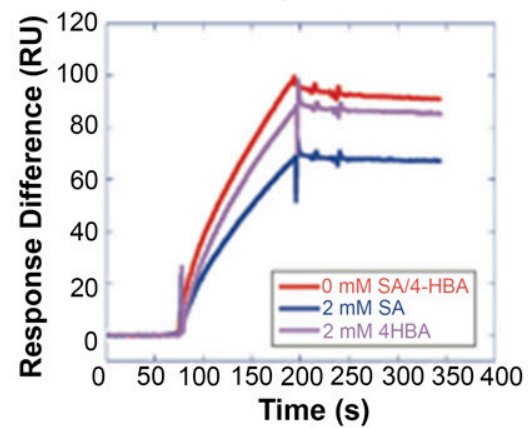

Fig. 3. Analysis of salicylic acid (SA)-binding activities of AtGAPC-1 and AtGAPC-2 via surface plasmon resonance. Sensograms of A and C, AtGAPC-1 $(3 \mu \mathrm{M})$ and $\mathbf{D}$ and $\mathbf{F}$, AtGAPC-2 $(2 \mu \mathrm{M})$ in the presence of the indicated concentrations of SA, 4-hydroxybenzoic acid (4-HBA), or equivalent volumes of $1 \times$ phosphate-buffered saline buffer. The mixture was either preincubated on ice for $1 \mathrm{~h}$ before being passed over the 3-AESA-immobilized sensor chip surface (A and D) or passed directly over the chip without preincubation (B, C, E, and F). The signal from the mock-immobilized surface was subtracted. 
preferentially bind viral (-)RNA is critical for the robust production of (+)RNA progeny (Wang and Nagy 2008). In this report, we demonstrate that AtGAPC-1, like its yeast ortholog, binds the (-)-strand of the DI-72 repRNA with high efficiency, whereas its interaction with the (+)-strand is weak. Although Arabidopsis is not a host for TBSV, the above findings strongly suggest that cytosolic GAPDHs from TBSV host plants also will exhibit conserved features with their Arabidopsis ortholog, including SA-binding activity, which could regulate their

\section{A Replication in yeast}
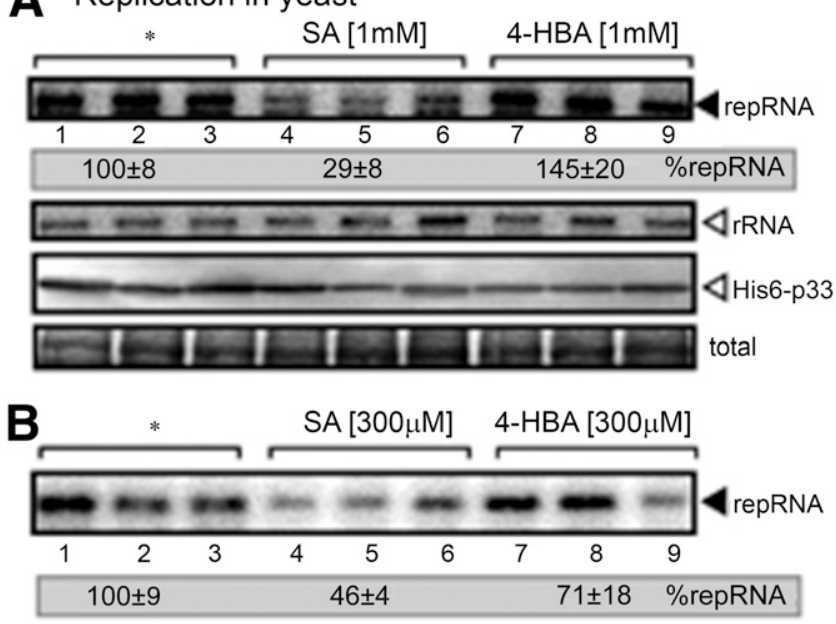

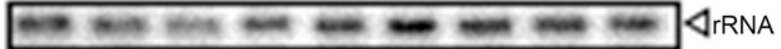

Fig. 4. Salicylic acid (SA) inhibits the replication of TBSV in vivo in yeast. Total RNA and protein extracts were prepared from yeast cells $24 \mathrm{~h}$ after induction of repRNA replication in the absence (*) or presence of $\mathbf{A}, 1 \mathrm{mM}$ or $\mathbf{B}, 300 \mu \mathrm{M}$ of SA or its biologically inactive analog 4-hydroxybenzoic acid (4-HBA). Northern blot analysis of total RNA extracts was done with a probe for DI-72(+), the TBSV replicon RNA (repRNA). Ribosomal RNA (rRNA) served as the loading control. The accumulation level of repRNA was normalized based on the rRNA. Immunoblot analysis with anti-His antibody was done to detect the level of $\mathrm{His}_{6}$-tagged $\mathrm{p} 33$, a protein required for tombusvirus replication. The assay confirmed that translation of p33 is not inhibited by SA. Total protein is shown as a loading control. Assays were done in triplicate, with the average level of viral RNA \pm standard deviation presented as a percentage of the replication observed in the absence of SA, which was set at $100 \%$. ability to promote TBSV replication. Our results are consistent with a previous report showing that acetylsalicylic acid induces resistance to TBSV in Gomphrena globosa plants (Pennazio and Redolfi 1980).

In addition to conferring TBSV resistance, the interaction between SA and GAPDHs may regulate other plant cellular processes. For example, SA binding might modulate the glycolytic activity of AtGAPC- 1 and AtGAPC- 2 or the carbon cycle activity of AtGAPA-1 and AtGAPA-2, thereby affecting carbon metabolism. Furthermore, the conversion of glyceraldehyde 3-phosphate to 1,3-bisphosphoglycerate during glycolysis involves the concomitant reduction of NAD+ to NADH (Rius et al. 2008), whereas the reverse reaction in the carbon cycle uses NADPH and generates NADP+ (Baalmann et al. 1995). Thus, SA binding to GAPDH might alter the NAD+/NADH ratio and, thereby, influence cellular redox. Because NADP+ buffers free radical formation by accepting electrons generated during photosynthesis (Price et al. 1995), inhibition of the GAPA isoforms by SA also might lead to increased production of reactive oxygen species (ROS). Interestingly, stress conditions that induce the accumulation of ROS, such as drought, cold, salinity, and elicitors of plant defense responses, inhibit the glycolytic activity of cytoplasmic GAPDH (GAPC) (Zaffagnini et al. 2013). Instead, these conditions promote various nonmetabolic (moonlighting) functions of GAPC, similar to those observed in animal homologs, via various redox-regulated posttranslational modifications such as sulfenation, glutathionylation, and S-nitrosylation, as well as nuclear localization (Zaffagnini et al. 2013). Recently, oxidation of GAPC also was shown to promote its ability to bind and stimulate the activity of a novel protein partner, the plasma membraneassociated phospholipase $\mathrm{D}$; this interaction appears to be involved in transducing the ROS signal initiated by drought stress (Guo et al. 2012). Whether SA binds and modulates these other moonlighting activities of GAPC is currently unknown.

In summary, this study has identified several members of the GAPDH protein family as SABPs. Although elucidating the physiological effects exerted when different GAPDH isoforms bind SA clearly will require further study, here we demonstrate that SA binding to AtGAPC-1 disrupts its ability to preferentially bind the (-)RNA of TBSV. Because this secondary activity has been co-opted to promote TBSV replication, our results reveal a novel molecular mechanism through which SA's antiviral activity can be mediated.

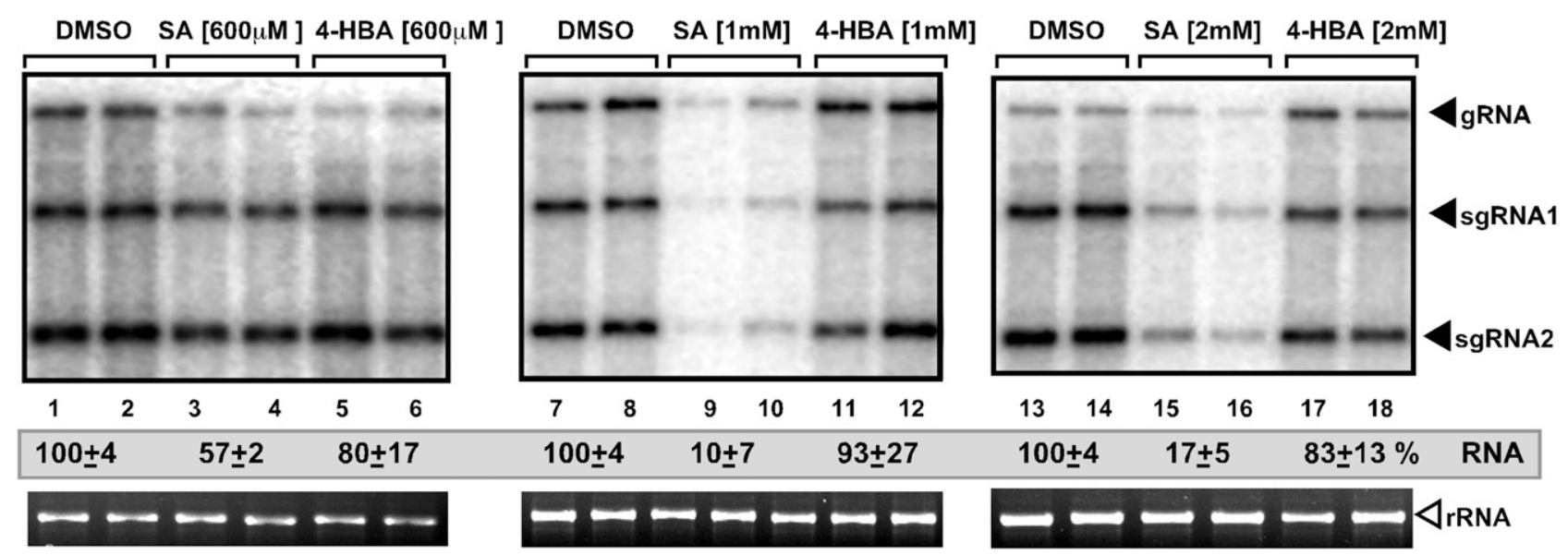

Fig. 5. Salicylic acid (SA) inhibits full-length TBSV RNA accumulation in Nicotiana benthamiana protoplasts. Total RNA was obtained from protoplasts 24 h after electroporation with infectious TBSV RNA present in total RNA prepared from TBSV-infected N. benthamiana. Electroporation of protoplasts was performed in the presence of $0,600 \mu \mathrm{M}, 1 \mathrm{mM}$, or $2 \mathrm{mM} \mathrm{SA}$ or 4-HBA. Top images: Northern blot analysis of total RNA extracts was done with a TBSV (+)RNAspecific probe. Bottom images: Ribosomal RNA (rRNA) served as the loading control. The accumulation level of TBSV RNAs was normalized based on the rRNA. 


\section{MATERIALS AND METHODS}

\section{Identification of putative SA-binding proteins from Arabidopsis.}

A photoaffinity labeling approach using the photoreactive SA analog 4-AzSA as a probe and anti-SA antibodies (AbD Serotec) (Tian et al. 2012) was utilized to identify cSABPs from Arabidopsis. Total proteins were extracted from the uninoculated distal leaves of Arabidopsis Col-0 plants $48 \mathrm{~h}$ postinoculation after primary infection with Pseudomonas syringae pv. maculicola ES4326 expressing AvrRpt2, and cross-linked with $0.5 \mathrm{mM}$ 4-AzSA. The cross-linked proteins were then pulled down with anti-SA antibodies after they were denatured in $4 \mathrm{M}$ urea to expose SA moiety of 4-AzSA from their binding pockets and make it fully accessible to the $\alpha$-SA Ab, followed by elution in $5 \mathrm{mM}$ SA. The isolated 4-AzSA-linked proteins were identified with tandem mass spectrometry, as described previously (Tian et al. 2012). A label-free semiquantitative proteomics approach was utilized to analyze all proteins from both the 4-AzSA cross-linked sample and the negative control sample. Proteins with a minimum of two spectral counts identified in the 4-AzSA cross-linked sample that were at least twofold more abundant than in the negative control or absent in the negative control were chosen as cSABPs.

Plasmid construction, protein expression, and purification.

The chloroplast transit peptides of AtGAPA-1, AtGAPA-2, and AtGAPB were predicted by ChloroP 1.1 and their coding segments were excluded from the expression plasmids. The

TBSV replication assay in yeast CFE:

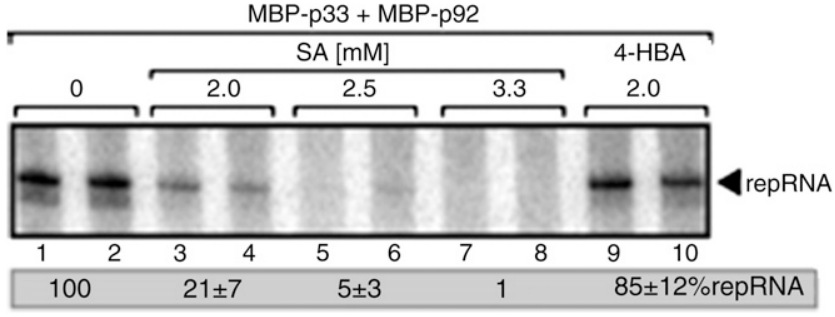

Fig. 6. Salicylic acid (SA) but not 4-hydroxybenzoic acid (4-HBA) inhibits the replication of TBSV in a yeast cell-free extract (CFE)-based assay. Purified recombinant MBP-p33, MBP-p92, and T7 transcripts of TBSV DI-72 (+) repRNA were added to yeast CFE together with isotope-labeled and unlabeled ribonucleotides. An increasing amount of SA or 4-HBA was added to the assay as indicated. The ${ }^{32} \mathrm{P}$-labeled repRNA products were analyzed on denaturing polyacrylamide gel. Assays were done in duplicate. plasmids pET28a-AtGAPA-1(46-396), pET28a-AtGAPA-2 (49-399), pET28a-AtGAPB(46-447), and pET28a-AtGAPC-2 were constructed by cloning polymerase chain reaction (PCR)amplified protein-encoding sequences minus the predicted transit peptides coding segment into BamHI and SalI sites of pET28a to express N-terminally His-tagged proteins in E. coli. pET28aAtGAPC-1 plasmids were constructed by PCR-amplified proteinencoding sequences into BamHI and HindIII sites of pET28a. The primers used were AtGAPA-1(46-396) (F: 5'-gcgggatccGC AATGGGAAGCAGTGGTGGATACAG-3' and R: 5' -gcggtcgac TCACTTCCAGTTGTTGGCAACAATG-3'), AtGAPA-2(49399) (F: 5'-gcgggatccGCAATGAGAAGCAATGGTGGATAC-3' and R: 5'-gcggtcgacTCACTTCCAGTTATTGGCAAC-3'), AtG APB(46-447) (F: 5'-gcgggatccATGAGTAGTATCGGTGGGGA AG-3' and R: 5' -gcggtcgacTCAGTCATAGACTTTGCATTCC T-3'), AtGAPC-1 (F: 5'-gcgggatccATGGCTGACAAGAAGAT TAGGATC-3' and R: 5'-gcgaagcttTTAGGCCTTTGACATGT GGACGATC-3'), and AtGAPC-2 (F: 5'-gcgggatccATGGCTG ACAAGAAGATCAGAATC-3' and R: 5' -geggtcgacTTAGGC CTTTGACATGTGAAC-3'). Gene-specific sequences are in uppercase letters and the restriction sites are underlined. The His-tagged recombinant proteins were expressed and purified in E. coli, as described previously (Kumar and Klessig 2003).

\section{SA-binding activity analysis.}

SA-binding activity analyses by photoaffinity labeling and SPR were performed as described previously (Tian et al. 2012).

\section{Analysis of the effect of SA on replication of TBSV DI-72 replicon RNA.}

Saccharomyces cerevisiae strain BY4741 (MATa his3 31 leu $2 \Delta 0$ met $15 \Delta 0$ ura3 $\Delta 0$ ) was transformed with plasmids pGBK-CUP1-6xHisp33/ADH1-DI-72 (his3 selection) (Mendu et al. 2010) and pGAD-CUP1-6xHisp92 (leu2 selection) (Serviene et al. 2006). Cells were grown at $23^{\circ} \mathrm{C}$ overnight in $\mathrm{SC}-\mathrm{LH}^{-}$media containing bathocuproinedisulphonate copper chelator at the concentration of $100 \mu \mathrm{M}$ to inhibit the expression of the CUPl promoter-driven viral proteins. The media also contained $1 \mathrm{mM}$ or $300 \mu \mathrm{M}$ SA or 4 -HBA. The control samples contained only the ethanol solvent. Then, BCS was washed out and $\mathrm{CuSO}_{4}$ was added to a final concentration of $50 \mu \mathrm{M}$ to induce the expression of the viral proteins. The cells were harvested after $24 \mathrm{~h}$ and total RNA was extracted and phenol/chloroform purified and precipitated. TBSV DI-72 replicon RNA(repRNA) accumulation was detected by Northern blot analysis with $3^{\prime}$-specific ${ }^{32} \mathrm{P}$-labeled probe to repRNA (Panavas and Nagy 2003). The expression of $\mathrm{His}_{6}$-p33 viral
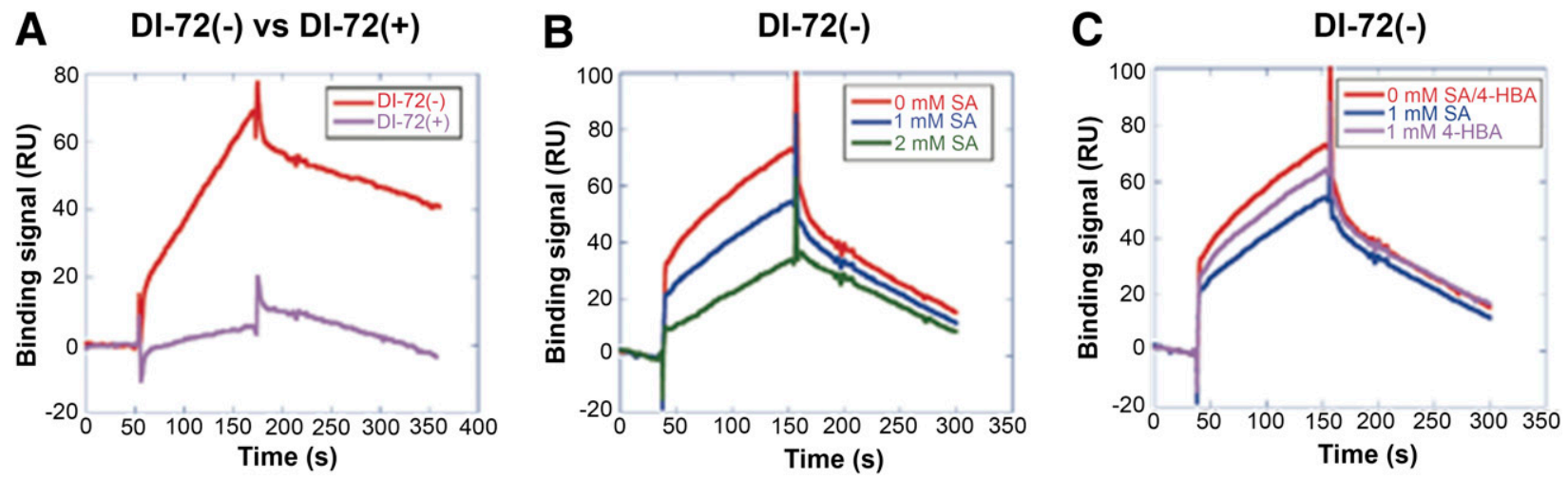

Fig. 7. Salicylic acid (SA) inhibits the binding of AtGAPC-1 to TBSV DI-72(-) repRNA. A, Sensograms were generated by passing DI-72(-) or Di-72(+) (1 $\mu$ M), in the absence or presence of the indicated concentrations of B, SA or C, 4-HBA, over an NTA sensor chip surface containing immobilized AtGAPC-1. The specific binding signal was determined by subtracting the signal generated with the control flow cell from the signal generated with the AtGAPC-1-bound flow cell. 
protein was analyzed by Western blot with $\mathrm{His}_{6}$-specific antibody.

Yeast CFEs capable of supporting TBSV replication in vitro were prepared as previously described (Pogany et al. 2008). SA or 4-HBA was added to the reactions to the final concentrations of $0,2,2.5$, and $3.3 \mathrm{mM}$. The assays were performed at $25^{\circ} \mathrm{C}$ for $3 \mathrm{~h}$. The newly synthesized ${ }^{32} \mathrm{P}$-labeled RNA products were separated by electrophoresis in a $5 \%$ polyacrylamide gel with $8 \mathrm{M}$ urea, containing $0.5 \times$ Tris-borate-EDTA buffer.

\section{Measuring the effect of SA on replication \\ of TBSV genomic RNA in $N$. benthamiana protoplasts.}

Isolation of protoplasts from $N$. benthamiana callus by treatment with $1 \mathrm{~g}$ of Cellulysin and $0.2 \mathrm{~g}$ of Macerase (Calbiochem) was performed as described previously (Panaviene et al. 2003). Protoplasts were electroporated with $2 \mu \mathrm{g}$ of a total RNA from TBSV-infected $N$. benthamiana obtained by the phenolchloroform method (Nagy et al. 2001). SA or 4-HBA were dissolved in dimethyl sulfoxide (DMSO) and were added at a final concentration of $0.6,1$, or $2 \mathrm{mM}$ a few seconds prior to electroporation. DMSO was used as a control applied in the same concentration as for SA or 4-HBA treatment. After electroporation, samples were kept on ice for $30 \mathrm{~min}$, followed by adding $1.7 \mathrm{ml}$ of protoplast culture medium (Kong et al. 1997) to each sample. Protoplasts were incubated in 35-by10 -mm petri dishes in the dark for $24 \mathrm{~h}$ at room temperature.

For extraction of total RNA, protoplasts were harvested by centrifugation with no breaks to avoid premature rupture. Cells were broken by vortexing for approximately $30 \mathrm{~s}$ with equal volumes of RNA extraction buffer (50 mM NaAc [pH 5.2], $10 \mathrm{mM}$ EDTA, and $1 \%$ sodium dodecyl sulfate) and watersaturated phenol followed by a 5-min centrifugation at maximum $\mathrm{rpm}$ at $4^{\circ} \mathrm{C}$. The aqueous phase was transferred to saturated phenol-chloroform (approximately $250 \mu \mathrm{l}$ in volume) mixed in a 1:1 ratio, vortexed, and centrifuged again for $5 \mathrm{~min}$ at maximum $\mathrm{rpm}$ at $4^{\circ} \mathrm{C}$. RNA was precipitated from the final aqueous phase in absolute ethanol at $-20^{\circ} \mathrm{C}$, washed with $70 \%$ ethanol, and dissolved in sterile water. The obtained RNA samples were separated on a $1.5 \%$ agarose gel and transferred to a Hybond-XL membrane (Amersham) before hybridization with a ${ }^{32} \mathrm{P}$-labeled TBSV DI-72-specific probe (Panavas and Nagy 2003). Viral RNA accumulation was normalized based on rRNA using the ImageQuant software and a Typhoon scanner (General Electric).

\section{In vitro transcription of DI-72(+) and DI-72(-) RNAs.}

DI-72(+) and DI-72(-) were synthesized by in vitro transcription using T7 RNA polymerase (New England Biolabs) following the manufacturer's instructions. DNA templates in the reaction mix were removed using RQ1 DNase (Promega). The synthesized RNAs were purified with a SurePrep RNA cleanup and concentration kit (Fisher Scientific).

\section{Binding analyses of AtGAPC-1 with DI-72 $(-)$ and DI-72(+).}

The binding analyses of AtGAPC-1 with DI-72(-) and DI-72(+) were performed by SPR using a Biacore 3000 instrument. Histagged AtGAPC-1 was immobilized on an NTA sensor chip (GE Healthcare), essentially following the manufacturer's instructions. HBS-P buffer (0.01 M HEPES [pH 7.4], $0.15 \mathrm{M}$ $\mathrm{NaCl}$, and $0.005 \%$ [vol/vol] Surfactant P20) supplemented with $50 \mu \mathrm{M}$ EDTA was used as the running buffer, and HBSEP buffer (0.01 M HEPES [pH 7.4], $0.15 \mathrm{M} \mathrm{NaCl}, 3 \mathrm{mM}$ EDTA, and $0.005 \%$ [vol/vol] Surfactant P20) was used as dispenser buffer. To immobilize His-AtGAPC-1, $10 \mu \mathrm{l}$ of $0.5 \mathrm{mM}$ $\mathrm{NiCl}_{2}$ (NTA reagent kit; GE Healthcare) was flowed over the NTA sensor chip surface, followed by $25 \mu \mathrm{l}$ of $0.5 \mu \mathrm{M}$ of HisAtGAPC-1. The immobilization level was stable under this condition. To test the binding of His-AtGAPC-1 with RNAs of DI-72(-) or DI-72(+) and the effect of SA on the binding, $20 \mu \mathrm{l}$ of $1 \mu \mathrm{M}$ RNAs in the absence or presence of various concentrations of SA or 4-HBA was flowed over both the AtGAPC-1immobilized flow cell and the nonimmobilized control flow cell. The specific binding signal was determined by subtracting the signal generated with the control flow cell from the signal generated with the AtGAPC-1-bound flow cell. To reuse the chip, bound proteins together with RNAs were stripped off by injecting $30 \mu \mathrm{l}$ of $350 \mathrm{mM}$ EDTA (NTA reagent kit, GE Healthcare). The flow rate used throughout the experiment was set at $10 \mu \mathrm{min}^{-1}$.

\section{ACKNOWLEDGMENTS}

We thank D. Dempsey for editing the manuscript. This work was supported by grants from the National Science Foundation to D. F. Klessig and K. J. van Wijk (IOS-0820405) and P. D. Nagy (MCB-1122039).

\section{LITERATURE CITED}

Alamillo, J. M., Saénz, P., and García, J. A. 2006. Salicylic acid-mediated and RNA-silencing defense mechanisms cooperate in the restriction of systemic spread of Plum pox virus in tobacco. Plant J. 48:217-227.

An, C., and Mou, Z. 2011. Salicylic acid and its function in plant immunity. J. Integr. Plant Biol. 53:412-428.

Baalmann, E., Backhausen, J. E., Rak, C., Vetter, S., and Scheibe, R. 1995. Reductive modification and nonreductive activation of purified spinach chloroplast NADP-dependent glyceraldehyde-3-phosphate dehydrogenase. Arch. Biochem. Biophys. 324:201-208.

Baebler, Š., Witek, K., Petek, M., Stare, K., Tušek-Žnidarič, M., PompeNovak, M., Renaut, J., Szajko, K., Strzelczyk-Żyta, D., Marczewski, W., Morgiewicz, K., Gruden, K., and Hennig, J. 2014. Salicylic acid is an indispensable component of the Ny-1 resistance-gene-mediated response against Potato virus $Y$ infection in potato. J. Exp. Bot. 65:1095-1109.

Carr, J. P., Lewsey, M. G., and Palukaitis, P. 2010. Signaling in induced resistance. Adv. Virus Res. 76:57-121.

Chen, Z., Silva, H., and Klessig, D. F. 1993. Active oxygen species in the induction of plant systemic acquired resistance by salicylic acid. Science 262:1883-1886.

Chisholm, S. T., Coaker, G., Day, B., and Staskawicz, B. J. 2006 Host-microbe interactions: Shaping the evolution of the plant immune response. Cell 124:803-814.

Chivasa, S., Murphy, A. M., Naylor, M., and Carr, J. P. 1997. Salicylic acid interferes with tobacco mosaic virus replication via a novel salicylhydroxamic acid-sensitive mechanism. Plant Cell 9:547-557.

De, B. P., Gupta, S., Zhao, H., Drazba, J. A., and Banerjee, A. K. 1996. Specific interaction in vitro and in vivo of glyceraldehyde-3-phosphate dehydrogenase and LA protein with cis-acting RNAs of human parainfluenza virus type 3. J. Biol. Chem. 271:24728-24735.

Dempsey, D. A., and Klessig, D. F. 2012. SOS-Too many signals for systemic acquired resistance? Trends Plant Sci. 17:538-545.

Durner, J., and Klessig, D. F. 1995. Inhibition of ascorbate peroxidase by salicylic acid and 2,6-dichloroisonicotinic acid, two inducers of plant defense responses. Proc. Natl. Acad. Sci. U.S.A. 92:11312-11316.

Durrant, W. E., and Dong, X. 2004. Systemic acquired resistance. Annu. Rev. Phytopathol. 42:185-209.

Fu, Z. Q., Yan, S., Saleh, A., Wang, W., Ruble, J., Oka, N., Mohan, R., Spoel, S. H., Tada, Y., Zheng, N., and Dong, X. 2012. NPR3 and NPR4 are receptors for the immune signal salicylic acid in plants. Nature 486:228-232.

Guo, L., Devaiah, S. P., Narasimhan, R., Pan, X., Zhang, Y., Zhang, W., and Wang X. 2012. Cytosolic glyceraldehyde-3-phosphate dehydrogenases interact with phospholipase $\mathrm{D} \delta$ to transduce hydrogen peroxide signals in the Arabidopsis response to stress. Plant Cell 24:2200-2212.

Huang, T. S., and Nagy, P. D. 2011. Direct inhibition of tombusvirus plusstrand RNA synthesis by a dominant negative mutant of a host metabolic enzyme, glyceraldehyde-3-phosphate dehydrogenase, in yeast and plants. J. Virol. 85:9090-9102.

Ji, L. H., and Ding, S. W. 2001. The suppressor of transgene RNA silencing encoded by Cucumber mosaic virus interferes with salicylic acidmediated virus resistance. Mol. Plant-Microbe Interact. 14:715-724

Jones, J. D., and Dangl, J. L. 2006. The plant immune system. Nature 444: 323-329.

Kong, Q., Wang, J., and Simon, A. E. 1997. Satellite RNA-mediated resistance to turnip crinkle virus in Arabidopsis involves a reduction in virus movement. Plant Cell 9:2051-2063. 
Kovalev, N., Pogany, J., and Nagy, P. D. 2012. A Co-Opted DEAD-Box RNA helicase enhances tombusvirus plus-strand synthesis. PLoS Pathog. 8:e1002537.

Kumar, D., and Klessig, D. F. 2003. High-affinity salicylic acid-binding protein 2 is required for plant innate immunity and has salicylic acidstimulated lipase activity. Proc. Natl. Acad. Sci. U.S.A. 100:16101-16106.

Liu, Y., Gao, Q., Wu, B., Ai, T., and Guo, X. 2009. NgRDR1, an RNAdependent RNA polymerase isolated from Nicotiana glutinosa, was involved in biotic and abiotic stresses. Plant Physiol. Biochem. 47:359-368.

Mendu, V., Chiu, M., Barajas, D., Li, Z., and Nagy, P. D. 2010. Cpr1 cyclophilin and Ess1 parvulin prolyl isomerases interact with the tombusvirus replication protein and inhibit viral replication in yeast model host. Virology 406:342-351.

Moreau, M., Westlake, T., Zampogna, G., Popescu, G., Tian, M., Noutsos, C., and Popescu, S. 2013. The Arabidopsis oligopeptidases TOP1 and TOP2 are salicylic acid targets that modulate SA-mediated signaling and the immune response. Plant J. 76:603-614.

Muñoz-Bertomeu, J., Cascales-Miñana, B., Mulet, J. M., Baroja-Fernández, E., Pozueta-Romero, J., Kuhn, J. M., Segura, J., and Ros, R. 2009. Plastidial glyceraldehyde-3-phosphate dehydrogenase deficiency leads to altered root development and affects the sugar and amino acid balance in Arabidopsis. Plant Physiol. 151:541-558.

Murphy, A. M., Chivasa, S., Singh, D. P., and Carr, J. P. 1999. Salicylic acid-induced resistance to viruses and other pathogens: A parting of the ways? Trends Plant Sci. 4:155-160.

Nagy, P. D., and Pogany, J. 2012. The dependence of viral RNA replication on co-opted host factors. Nat. Rev. Microbiol. 10:137-149.

Nagy, P. D., Pogany, J., and Simon, A. E. 2001. In vivo and in vitro characterization of an RNA replication enhancer in a satellite RNA associated with Turnip crinkle virus. Virology 288:315-324.

Panavas, T., and Nagy, P. D. 2003. Yeast as a model host to study replication and recombination of defective interfering RNA of Tomato bushy stunt virus. Virology 314:315-325.

Panaviene, Z., Baker, J. M., and Nagy, P. D. 2003. The overlapping RNAbinding domains of $\mathrm{p} 33$ and $\mathrm{p} 92$ replicase proteins are essential for tombusvirus replication. Virology 308:191-205.

Pennazio, S., and Redolfi, P. 1980. Resistance to tomato bushy stunt virus localised infection induced in Gomphrena globosa by acetylsalicylic acid. Microbiologica 3:475-479.

Petrik, J., Parker, H., and Alexander, G. J. 1999. Human hepatic glyceraldehyde-3-phosphate dehydrogenase binds to the poly(U) tract of the 3 ' non-coding region of hepatitis $\mathrm{C}$ virus genomic RNA. J. Gen. Virol. 80:3109-3113.

Pogany, J., Stork, J., Li, Z., and Nagy, P. D. 2008. In vitro assembly of the Tomato bushy stunt virus replicase requires the host Heat shock protein 70. Proc. Natl. Acad. Sci. U.S.A. 105:19956-19961.

Price, G. D., Evans, J. R., von Caemmerer, S., Yu, J. W., and Badger, M. R. 1995. Specific reduction of chloroplast glyceraldehyde-3-phosphate dehydrogenase activity by antisense RNA reduces $\mathrm{CO}_{2}$ assimilation via a reduction in ribulose bisphosphate regeneration in transgenic tobacco plants. Planta 195:369-378.

Quilis, J., Peñas, G., Messeguer, J., Brugidou, C., and San Segundo, B. 2008. The Arabidopsis AtNPR1 inversely modulates defense responses against fungal, bacterial, or viral pathogens while conferring hypersensitivity to abiotic stresses in transgenic rice. Mol. Plant-Microbe Interact. 21:1215-1231.

Rius, S. P., Casati, P., Iglesias, A. A., and Gomez-Casati, D. F. 2008. Characterization of Arabidopsis lines deficient in GAPC-1, a cytosolic NAD-dependent glyceraldehyde-3-phosphate dehydrogenase. Plant Physiol. 148:1655-1667.

Sánchez, G., Gerhardt, N., Siciliano, F., Vojnov, A., Malcuit, I., and Marano, M. R. 2010. Salicylic acid is involved in the Nb-mediated defense responses to Potato virus $X$ in Solanum tuberosum. Mol. PlantMicrobe Interact. 23:394-405.

Schultz, D. E., Hardin, C. C., and Lemon, S. M. 1996. Specific interaction of glyceraldehyde 3-phosphate dehydrogenase with the 5'-nontranslated RNA of hepatitis A virus. J. Biol. Chem. 271:14134-14142.

Serva, S., and Nagy, P. D. 2006. Proteomics analysis of the tombusvirus replicase: Hsp70 molecular chaperone is associated with the replicase and enhances viral RNA replication. J. Virol. 80:2162-2169.

Serviene, E., Jiang, Y., Cheng, C. P., Baker, J., and Nagy, P. D. 2006. Screening of the yeast yTHC collection identifies essential host factors affecting tombusvirus RNA recombination. J. Virol. 80:1231-1241.
Singh, D. P., Moore, C. A., Gilliland, A., and Carr, J. P. 2004. Activation of multiple antiviral defence mechanisms by salicylic acid. Mol. Plant Pathol. 5:57-63.

Sirover, M. A. 1999. New insights into an old protein: The functional diversity of mammalian glyceraldehyde-3-phosphate dehydrogenase. Biochim. Biophys. Acta 1432:159-184.

Sirover, M. A. 2005. New nuclear functions of the glycolytic protein, glyceraldehyde-3-phosphate dehydrogenase, in mammalian cells. J. Cell. Biochem. 95:45-52.

Slaymaker, D. H., Navarre, D. A., Clark, D., del Pozo, O., Martin, G. B., and Klessig, D. F. 2002. The tobacco salicylic acid-binding protein 3 (SABP3) is the chloroplast carbonic anhydrase, which exhibits antioxidant activity and plays a role in the hypersensitive defense response. Proc. Natl. Acad. Sci. U.S.A. 99:11640-11645.

Soosaar, J. L., Burch-Smith, T. M., and Dinesh-Kumar, S. P. 2005. Mechanisms of plant resistance to viruses. Nat. Rev. Microbiol. 3: 789-798.

Takahashi, H., Miller, J., Nozaki, Y., Sukamto,Takeda, M., Shah, J., Hase, S., Ikegami, M., Ehara, Y., and Dinesh-Kumar, S. P. 2002. RCY1, an Arabidopsis thaliana RPP8/HRT family resistance gene, conferring resistance to Cucumber mosaic virus requires salicylic acid, ethylene and a novel signal transduction mechanism. Plant J. 32:655-667.

Tian, M., von Dahl, C. C., Liu, P. P., Friso, G., van Wijk, K. J., and Klessig, D. F. 2012. The combined use of photoaffinity labeling and surface plasmon resonance-based technology identifies multiple salicylic acidbinding proteins. Plant J. 72:1027-1038.

Tristan, C., Shahani, N., Sedlak, T. W., and Sawa, A. 2011. The diverse functions of GAPDH: Views from different subcellular compartments. Cell. Signal. 23:317-323.

Vlot, A. C., Liu, P. P., Cameron, R. K., Park, S. W., Yang, Y., Kumar, D., Zhou, F., Padukkavidana, T., Gustafsson, C., Pichersky, E., and Klessig, D. F. 2008. Identification of likely orthologs of tobacco salicylic acidbinding protein 2 and their role in systemic acquired resistance in Arabidopsis thaliana. Plant J. 56:445-456.

Vlot, A. C., Dempsey, D. A., and Klessig, D. F. 2009. Salicylic Acid, a multifaceted hormone to combat disease. Annu. Rev. Phytopathol. 47: 177-206.

Wang, R. Y., and Nagy, P. D. 2008. Tomato bushy stunt virus co-opts the RNA-binding function of a host metabolic enzyme for viral genomic RNA synthesis. Cell Host Microbe 3:178-187.

White, K. A., and Nagy, P. D. 2004. Advances in the molecular biology of tombusviruses: Gene expression, genome replication, and recombination. Prog. Nucleic Acid Res. Mol. Biol. 78:187-226.

Wu, Y., Zhang, D., Chu, J. Y., Boyle, P., Wang, Y., Brindle, I. D., De Luca, V., and Després, C. 2012. The Arabidopsis NPR1 protein is a receptor for the plant defense hormone salicylic acid. Cell Reports 1: 639-647.

Xie, Z., Fan, B., Chen, C., and Chen, Z. 2001. An important role of an inducible RNA-dependent RNA polymerase in plant antiviral defense. Proc. Natl. Acad. Sci. U.S.A. 98:6516-6521.

Yang, S. J., Carter, S. A., Cole, A. B., Cheng, N. H., and Nelson, R. S. 2004 A natural variant of a host RNA-dependent RNA polymerase is associated with increased susceptibility to viruses by Nicotiana benthamiana. Proc. Natl. Acad. Sci. U.S.A. 101:6297-6302.

Yu, D., Fan, B., MacFarlane, S. A., and Chen, Z. 2003. Analysis of the involvement of an inducible Arabidopsis RNA-dependent RNA polymerase in antiviral defense. Mol. Plant-Microbe Interact. 16: 206-216.

Zaffagnini, M., Fermani, S., Costa, A., Lemaire, S. D., and Trost, P. 2013. Plant cytoplasmic GAPDH: Redox post-translational modifications and moonlighting properties. Front. Plant Sci. 4:450.

Zang, W. Q., Fieno, A. M., Grant, R. A., and Yen, T. S. 1998. Identification of glyceraldehyde-3-phosphate dehydrogenase as a cellular protein that binds to the hepatitis B virus posttranscriptional regulatory element. Virology 248:46-52.

\section{AUTHOR-RECOMMENDED INTERNET RESOURCES}

The Arabidopsis Information Resource: www.arabidopsis.org AbD Serotec: www.abdserotec.com/

ChloroP 1.1: www.cbs.dtu.dk/services/ChloroP/ 